\title{
Zum 60. Geburtstag von Prof. Dr. med. Bernd Hamm
}

On the Occasion of the 60th Birthday of Prof. Dr. med. Bernd Hamm

M. Dewey, P. Asbach, U. Bick, B. Gebauer, T. Kröncke, M. Taupitz

Institut

Radiologie, Charité - Universitätsmedizin Berlin, Humboldt Universität und Freie Universität zu Berlin

Bibliografie

Dol http://dx.doi.org/10.1055/

s-0033-1350222

Fortschr Röntgenstr 2013; 185 : 810-811 @ Georg Thieme Verlag KG Stuttgart · New York · ISSN 1438-9029

Korrespondenzadresse

Prof. Marc Dewey

Klinik für Radiologie, Charité Universitätsmedizin Berlin, Humboldt Universität und Freie Universität zu Berlin

Charitéplatz 1

10117 Berlin

Tel.: ++49/30/450527296

Fax: $++49 / 30 / 450527996$

dewey@charite.de
Es ist uns eine große Freude, zahlreiche Beiträge dieser und folgender Ausgaben der Röfo unserem akademischen und klinischen Lehrer Herrn Professor Bernd Hamm anlässlich seines 60 . Geburtstags zu widmen ( $\boldsymbol{O}$ Abb. 1). Bernd Hamm wurde am 30.8.1953 in Frankfurt am Main geboren. Nach dem Studium der Humanmedizin an der Freien Universität Berlin von 1972- 1978 begann er seine ärztliche Laufbahn 1978 im Institut für Pathologie des Klinikums Steglitz der Freien Universität Berlin, wo er 1982 mit magna cum laude mit einem tierexperimentellen Thema promoviert wurde. Die Radiologie fesselte ihn jedoch stärker, so dass er 1980 die Weiterbildung zum Facharzt für Radiologie an der Klinik von Professor Karl-Jürgen Wolf begann und 1986 erfolgreich abschloss. Früh beschäftigte er sich mit der urogenitalen Diagnostik und publizierte hierzu 1991 sein erstes Lehrbuch über „Sonographische Diagnostik des Skrotalinhalts." Seit Mitte der 1980er-Jahre galt sein wissenschaftliches Interesse der Magnetresonanztomografie und den damals neu entwickelten paramagnetischen Kontrastmitteln, ein Gebiet, das Grundlage seiner Habilitation im Jahr 1989 wurde.

Von 1990 - 1993 war Bernd Hamm als Leitender Oberarzt an der Klinik von Professor Karl-Jürgen Wolf tätig und wurde 1993 auf eine C3-Professur für Klinische Radiologie an die Freie Universität Berlin berufen. Bernd Hamm nahm 1994 als Nachfolger von Professor Meinhard Lüning und dem kommissarischen Direktor Professor Walter Reisinger den Ruf auf die C4-Professur für Röntgendiagnostik verbunden mit der Leitung des Instituts für Radiologie der Charité der HumboldtUniversität zu Berlin (heute: Charité Campus Mitte) an. Mit Annahme des Rufes setzte Bernd Hamm zahlreiche neue Impulse. Neben klinischen Schwerpunkten baute er eine leistungsfähige experimentelle Forschung auf. Beispiele hierfür sind eine DFG-geförderte Emmy-Noether Nachwuchsgruppe zur „MR-Elastografie“ 2004, die DFG-geförderte Klinische Forschergruppe 213 zum Thema „Magnetische Eisenoxid-Nanopartikel für die Zelluläre und Molekulare MRBildgebung“ 2008 und zwei DFG-geförderte Heisenberg-Professuren zur MR-Elastografie seit 2010 und für nicht invasive kardiovaskuläre Bildgebung seit 2013. Auch die anwendungsorientierte Forschung wurde von Bernd Hamm im Blick behalten. In der Arbeitsgruppe experimen- telle Radiologie wurde unter anderem das Prinzip des Paclitaxel-beschichteten Ballonkatheters zur Behandlung von arteriellen Restenosen entwickelt, das derzeit eines der wichtigsten Patente der Charité darstellt.

Seit der Bildung von Centren innerhalb der über 3 Standorte fusionierten Charité in 2006 ist Bernd Hamm der Leiter des CharitéCentrums 6 für Diagnostische und Interventionelle Radiologie, Nuklearmedizin und Medizinische Physik. Im Jahr 2006 übernahm Bernd Hamm nach Emeritierung von Professor Roland Felix die Leitung der Klinik für Strahlenheilkunde am Campus Virchow-Klinikum der Charité und nach Emeritierung von Professor K.-J. Wolf im Jahr 2010 zusätzlich die Leitung der Klinik und Hochschulambulanz für Radiologie am Campus Benjamin Franklin der Charité. Mit großem Engagement trieb Bernd Hamm die weitere Verzahnung der drei radiologischen Standorte voran. Hierbei lag ihm der persönliche und wissenschaftliche Austausch der Mitarbeiter genauso am Herzen wie die Weiterentwicklung der Forschungsschwerpunkte am jeweiligen Campus. Die akademische Erfolgsbilanz ist mit 27 Habilitationen, 13 Chefarzternennungen und 14 Berufungen seit 2006 ein Spiegel seines unermüdlichen Ansporns seiner Mitarbeiterinnen und Mitarbeiter.

Bernd Hamm ist seit 2005 als Mitherausgeber der Röfo tätig [1]. In der Zeit von 2009-2012 war er deren federführender Herausgeber [2]. Bernd Hamm ist Mitherausgeber des 2007 erschienenen und in 7 Sprachen übersetzten Buches „Urogenitales System“ der Pareto-Reihe des Thieme-Verlags. Er ist Mitherausgeber des Standardwerks „MRT von Abdomen und Becken“ und außerdem fungiert er gemeinsam mit Professor Pablo Ros als Herausgeber der neuen Buchreihe „Abdominal Imaging“. 
Die klinische Bedeutung unseres Fachgebiets erklärt seine Begeisterung und sein vielfältiges Engagement für die Radiologie. Anlässlich der Hundertjahrfeier der Deutschen Röntgengesellschaft im Schlüterhof des Deutschen Historischen Museums in Berlin wies er in Bezug auf den ersten Röntgenkongress 1905 in Berlin auf das breite, interdisziplinäre Fundament der Radiologie hin, das durch neue bildgebende und interventionelle Verfahren deutlich ausgebaut worden ist [3]. Trotz seiner zahlreichen klinikinternen Verpflichtungen widmet sich Bernd Hamm auch intensiv berufspolitischen Aufgaben. Als 1. Vorsitzender der Berliner Röntgen-Gesellschaft/Röntgenvereinigung $\mathrm{zu}$ Berlin und Brandenburg (1995-1996) und als Präsident der Deutschen Röntgengesellschaft (2003-2005) setzte er sich sehr erfolgreich für berufspolitische Belange der Radiologie ein. Derzeit leitet er in der Deutschen Röntgengesellschaft die Arbeitsgruppe „Weiterbildungsordnung“ und bringt sich in die Novellierung der Musterweiterbildungsordnung ein. Des Weiteren ist Bernd Hamm 1. stellvertretender Vorsitzender im Berufsverband der Deutschen Radiologen. Nach gemeinsamer Präsidentschaft des DeutschÖsterreichischen Röntgenkongresses in 2011 mit Professor Walter Hruby aus Wien [4 - 7] wird Bernd Hamm 2015 der Präsident des European Congress of Radiology sein. Die Ehrenmitgliedschaften der Japanischen [8], Österreichischen und Schweizerischen Röntgengesellschaft sind Anerkennungen seiner Arbeit ebenso wie eine Ehrenprofessur des China-Japan Friendship Hospital in Peking und die Wahl zum Mitglied der Deutschen Akademie der Naturforscher Leopoldina.

Als besondere Eigenschaft von Bernd Hamm soll an dieser Stelle sein politisches und organisatorisches Talent gewürdigt werden, mit dem er die Berliner Universitätsradiologie äußerst erfolgreich durch eine Kaskade von Umbrüchen geführt hat. Hier sei besonders auf die Fusion der Charité mit dem Virchow-Klinikum und schließlich auch mit dem Klinikum Benjamin Franklin mit der Bildung einer campusübergreifenden Berliner Universitätsradiologie unter schwierigen wirtschaftlichen Rahmenbedingungen hingewiesen. Gleichzeitig hat Bernd Hamm die gesamte Klinik zu wissenschaftlicher und klinischer Exzellenz geführt und mit über 400 Mitarbeitern internationale Akzente gesetzt.

Im Namen seiner Kollegen, Schüler und Mitarbeiter gratulieren wir ihm sehr herzlich zum 60. Geburtstag und wünschen weiterhin viel Glück, Gesundheit, Schaffenskraft und Erfolg bei der Verwirklichung seiner Ziele in der Zukunft.

Der Georg Thieme Verlag, die RöFo-Mitwirkenden und -Herausgeber schließen sich diesen Glückwünschen an.

Marc Dewey, Patrick Asbach, Ulrich Bick, Bernhard Gebauer, Thomas Kröncke, Matthias Taupitz im Namen aller Mitarbeiterinnen und Mitarbeiter der Radiologie der Charité.

\section{Literatur}

1 Günther RW, Thelen $M$, Wolf KJ. Herausgeberwechsel bei der RöFo. Fortschr Röntgenstr 2005; 177: 157-158

2 Hamm B, Diekmann F. Rückblick und Dank für drei erfolgreiche Jahre. Fortschr Röntgenstr 2012; 184: 694-696

3 [Anonym]. „Nach dem Kongress ist vor dem Kongress“ Rückblick auf den 86. Deutschen Röntgenkongress vom 4. bis 7. Mai 2005 in Berlin; 2005, http://apps.drg.de/data/Roekon05 / Rueck2005.htm

4 [Anonym]. In Hamburg angekommen - Nachbericht zum 92. Deutschen Röntgenkongress, 6. gemeinsamer Kongress von DRG und ÖRG. Fortschr Röntgenstr 2011; 183: 769

5 Hamm B, Hruby W. Röntgenkongress 2011 in Hamburg. Fortschr Röntgenstr 2011; 183: 333

6 Hamm B, Hruby W. Interview - Radiologie ist ein Ganzkörperfach. Fortschr Röntgenstr 2011; 183: 397-398

7 Hruby W, Hamm B. 92. Deutscher Röntgenkongress - 6. Gemeinsamer Kongress von DRG und ÖRG - Radiologie trifft... Allgemeinmedizin Radiologen treffen Kollegen. Fortschr Röntgenstr 2011; 183: 485

8 [Anonym]. Ehrenmitgliedschaft - Japanische Röntgengesellschaft ehrt Prof. Hamm. Fortschr Röntgenstr 2010; 182: 443 